\title{
Laguerre-Gaussian Mode Laser Heater for Microbunching Instability Suppression in Free-Electron Lasers
}

\author{
Jingyi Tang $\odot,{ }^{1,2}$ Randy Lemons, ${ }^{1,3}$ Wei Liu, ${ }^{1}$ Sharon Vetter, ${ }^{1}$ Timothy Maxwell, ${ }^{1}$ Franz-Josef Decker, ${ }^{1}$ Alberto Lutman $\odot,{ }^{1}$ \\ Jacek Krzywinski, ${ }^{1}$ Gabriel Marcus, ${ }^{1}$ Stefan Moeller®, ${ }^{1}$ Zhirong Huang, ${ }^{1,2}$ Daniel Ratner, ${ }^{1}$ and Sergio Carbajo ${ }^{1,3, *}$ \\ ${ }^{1}$ SLAC National Accelerator Laboratory, 2575 Sand Hill Road, Menlo Park, California 94025, USA \\ ${ }^{2}$ Department of Applied Physics, Stanford University, Stanford, California 94305, USA \\ ${ }^{3}$ Colorado School of Mines, 1500 Illinois Street, Golden, Colorado 80401, USA
}

(Received 28 October 2019; revised manuscript received 29 January 2020; accepted 26 February 2020; published 30 March 2020)

\begin{abstract}
Microbunching instability (MBI) driven by beam collective effects is known to be detrimental to highbrightness storage rings, linacs, and free-electron lasers (FELs). One known way to suppress this instability is to induce a small amount of energy spread to an electron beam by a laser heater. The distribution of the induced energy spread greatly affects MBI suppression and can be controlled by shaping the transverse profile of the heater laser. Here, we present the first experimental demonstration of effective MBI suppression using a $\mathrm{LG}_{01}$ transverse laser mode and compare the improved results with respect to traditional Gaussian transverse laser mode at the Linac Coherent Light Source. The effects on MBI suppression are characterized by multiple downstream measurements, including longitudinal phase space analysis and coherent radiation spectroscopy. We also discuss the role of $\mathrm{LG}_{01}$ shaping in soft x-ray selfseeded FEL emission, one of the most advanced operation modes of a FEL for which controlled suppression of MBI is critical.
\end{abstract}

DOI: 10.1103/PhysRevLett.124.134801

Introduction.-Free-electron lasers (FELs) provide ultrashort, extremely bright coherent radiation at wavelengths down to the angstrom level and have been demonstrated to be powerful experimental tools in condensed matter physics, chemistry, and structural biology [1-6]. FELs require relativistic electron beams with high brightness and high current, which are generally achieved by magnetic compression along the accelerator [7]. During such process, the electron-beam ( $e$-beam) quality can be degraded due to microbunching instability (MBI) caused by collective effects like longitudinal space charge [8,9] and coherent synchrotron radiation [10-14], which are extensively studied both in theory and in experiment [15-19]. This instability amplifies the $e$-beam energy and density modulation and increases its energy spread up to a level that can be detrimental not only to the FEL gain process $[9,20]$, but also to the beam brightness in storage rings and linacs $[21,22]$.

MBI can be suppressed by a laser heater (LH) [8], which was first installed at the Linac Coherent Light Source (LCLS) and has been widely used in other FEL facilities $[9,15,20,23]$. The heater consists essentially of a short undulator within a chicane and a copropagating infrared

Published by the American Physical Society under the terms of the Creative Commons Attribution 4.0 International license. Further distribution of this work must maintain attribution to the author(s) and the published article's title, journal citation, and DOI.
(IR) laser that modulates and increases the energy spread of the $e$ beam by about one order of magnitude and without exceeding FEL tolerances. This increase in energy spread suppresses downstream MBI accumulation via Landau damping and has shown to result in a greater FEL intensity by factor of 3 [15].

The effectiveness of MBI suppression greatly depends not only on the amount of LH-induced energy spread but also on its distribution [9,24]. Each electron obtains a certain amplitude of energy modulation according to its relative location on the laser transverse distribution. Therefore, the induced energy spread distribution can be controlled by transversely shaping the LH pulse. The current LH at LCLS uses a simple transverse Gaussian-shaped laser matched to transverse $e$-beam shape. In the optimal condition when the spot size of the laser and $e$ beam are comparable, the induced energy distribution is of parabolic shape. In practice to fight the transverse jitter, the spot size of the laser is bigger than the $e$ beam and as a result double-horn energy distribution is induced. It is not most efficient in MBI suppression since the double horns can be regarded as two separate unheated cold beams $[9,15]$. Recent theoretical studies have investigated cylindrically symmetric and other nonconventional laser beam shapes to provide better suppression of microbunching [24,25]. In particular, a transverse Laguerre-Gaussian 01 $\left(\mathrm{LG}_{01}\right)$ mode provides Gaussian-shaped energy distribution and thus results in an exponential suppression in microbunching gain [24,25]. 


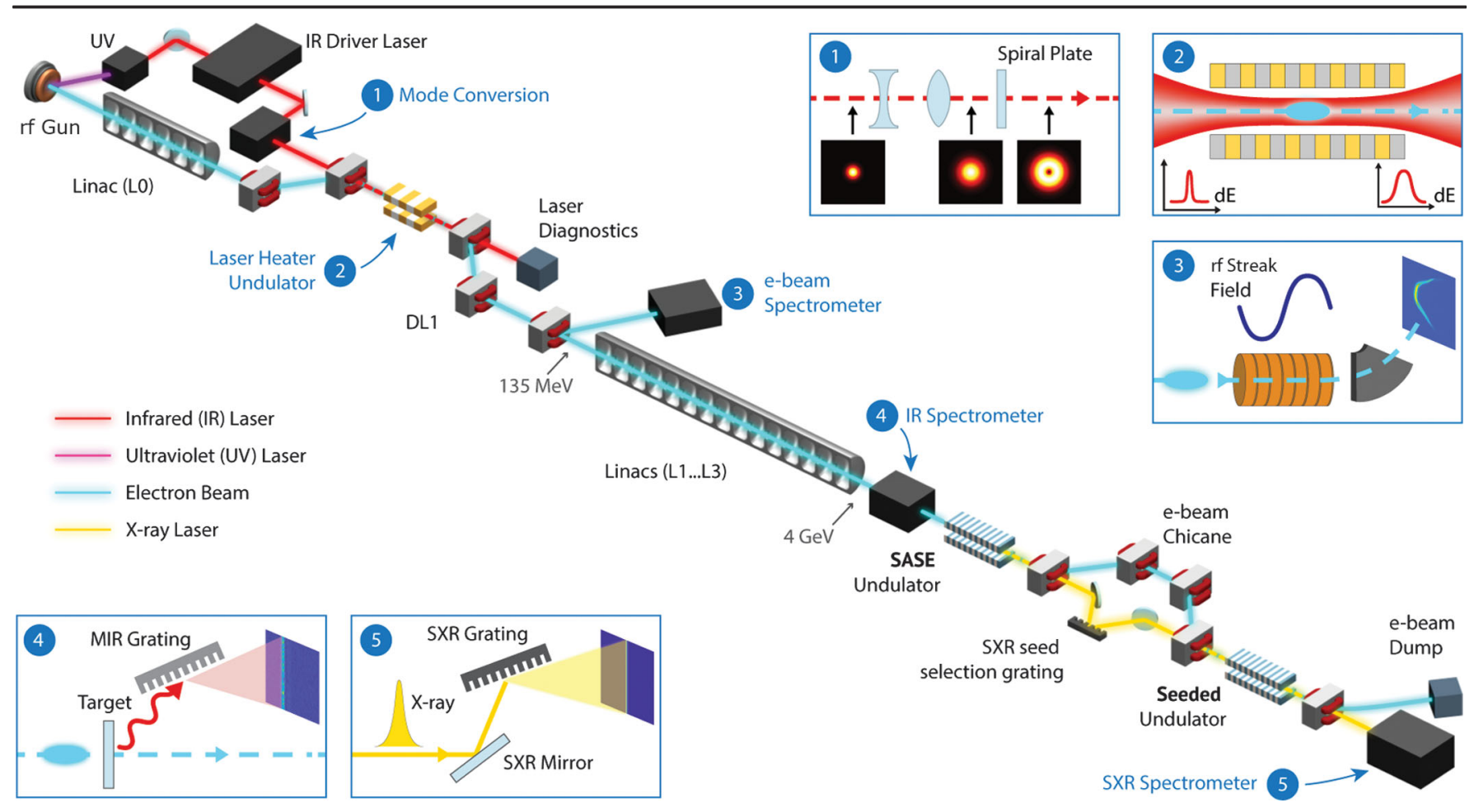

FIG. 1. Simplified schematic of start-to-end experimental configuration, from the photoinjector to the SXRSS diagnostic end station (not drawn to scale).

In this Letter, we investigate the use of a $\mathrm{LG}_{01}$ mode laser at the LCLS and its influence in MBI suppression and FEL performance. As our primary result, we demonstrate experimentally that a Gaussian-shaped energy distribution is induced by $\mathrm{LG}_{01}$ mode $\mathrm{LH}$ and final microbunching gain is better suppressed. We finally discuss the role of LH spatial shaping in soft x-ray self-seeded (SXRSS) FEL emission and demonstrate that this LH configuration is capable of generating clean, high spectral brightness FEL pulses, which is particularly critical for photon-hungry spectroscopies requiring careful selection of various elementary excitations [26].

Methods.-The layout of the optical mode conversion and downstream measurements are shown in Fig. 1. The LH transverse profile, originally Gaussian at LCLS, was converted to a $\mathrm{LG}_{01}$ distribution using a spiral phase plate (SPP) (inset 1 of Fig. 1). The SPP writes an increasing spiral phase onto the beam for a total phase change of $2 \pi$ and generates a null in the field amplitude at the center of the laser, as shown in Fig. 1, inset 2 [27].

For a $\mathrm{LG}_{01}$ mode laser heater, the $e$ beam should be located in the center of the laser, where it experiences a linear electric field that maps the Gaussian transverse distribution of the $e$ beam to its longitudinal plane. As a result, only a very small fraction of the laser power is effectively utilized. This entails that the power of the $\mathrm{LG}_{01}$ laser needs to be high enough to induce sufficient energy spread. In our experiments, the SPP enables over 95\% transmission efficiency and achieves laser energy of $\mathrm{LG}_{01}$ mode up to $1.8 \mathrm{~mJ}$, which is sufficient to induce energy spreads at the optimal level and higher. Table I lists the main parameters of the system.

Results.-To characterize the $\mathrm{LG}_{01}$ mode laser induced energy spread, the first diagnostic is directly downstream of the LH with a $135 \mathrm{MeV}$ spectrometer in Dogleg DL1 [15] (Fig. 1, inset 3). The dispersion in the spectrometer is $0.88 \mathrm{~m}$, corresponding to a resolution of $2.9 \mathrm{keV} /$ pixel. The slice energy distribution is extracted from the central time slice of the streaked electron beam. For either transverse shape, the width of the energy distribution can be tuned by varying the IR laser energy. Figure 2 shows the $e$ beam heated up to about $65 \mathrm{keV}$ energy spread at maximum laser energy. Since optimal MBI suppression typically requires $20-30 \mathrm{keV}$ induced energy spread, this constitutes enough range to study the effects of underheating and overheating in FEL performance. The induced energy spread is well fitted by a root curve relative to the laser energy and trickle heating effects at low energy is observed [15].

TABLE I. Laser heater parameters.

\begin{tabular}{lcl}
\hline \hline Parameter & Symbol & Value \\
\hline Electron energy at LH & $\gamma_{0} m c^{2}$ & $135 \mathrm{MeV}$ \\
LH undulator strength parameter & $K$ & 1.56 \\
LH undulator length & $L_{u}$ & $0.5 \mathrm{~m}$ \\
Initial peak current & $I_{0}$ & $30 \mathrm{~A}$ \\
Transverse electron beam size & $\sigma_{x}$ & $50 \mu \mathrm{m}$ \\
Transverse Gaussian mode laser size & $\sigma_{r G}$ & $100 \mu \mathrm{m}$ \\
Transverse $\mathrm{LG}_{01}$ mode laser size & $\sigma_{r}$ & $325 \mu \mathrm{m}$ \\
\hline \hline
\end{tabular}



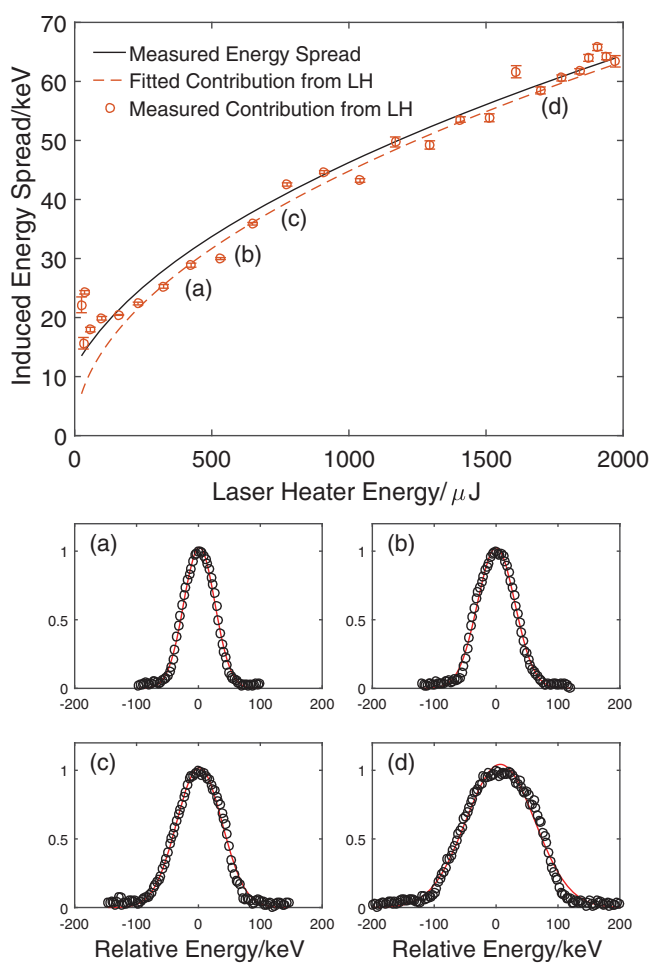

FIG. 2. Slice energy spread of the $e$ beam after the laser heater as a function of $\mathrm{LG}_{01}$ laser energy. Total energy spread measured at $135 \mathrm{MeV}$ spectrometer is fitted by the black line. The initial energy spread (with laser heater off) is removed in quadrature to obtain the red dashed line and the circles. (a)-(d) Four examples of energy distributions with (a) 25.1, (b) 30.3, (c) 36.8, and (d) $55.7 \mathrm{keV} \mathrm{rms}$ energy spread and their corresponding Gaussian fits.

Figures 2(a)-2(d) show typical structures of the induced energy spread distributions in circles and their corresponding Gaussian fitting in lines. The $\mathrm{LG}_{01}$ mode laser heater preserves the Gaussian-shaped distribution as the laser power increases, which is in good agreement with the theory [27]. For comparison, Figs. 3(a)-3(d) show the measured Gaussian mode laser heater induced energy distribution at different induced energy spread. As energy spread increases, the distributions clearly display a doublehorn structure, which is also consistent with previous analyses and measurements [15]. At low laser energy, the $e$-beam energy distribution looks Gaussian [Fig. 3(a)] due to the small induced energy spread and the limited resolution of the spectrometer.

To quantify the $\mathrm{LG}_{01}$ to Gaussian comparison, we compare the Gaussian $R^{2}$ fitting coefficient of both modes, averaged over 10 shots with highest $R^{2}$ at each laser energy, as shown in Fig. 3. It verifies that the $\mathrm{LG}_{01}$ mode laser consistently induces more Gaussian-shaped energy distribution. As its energy increases, the average $R^{2}$ of the $\mathrm{LG}_{01}$ mode decreases about $1 \%$ due to laser transverse jitter, which will be discussed later. We note that when the $e$ beam is heated to $20-30 \mathrm{keV}$, resembling optimal LCLS operation conditions, the Gaussian energy distribution is the
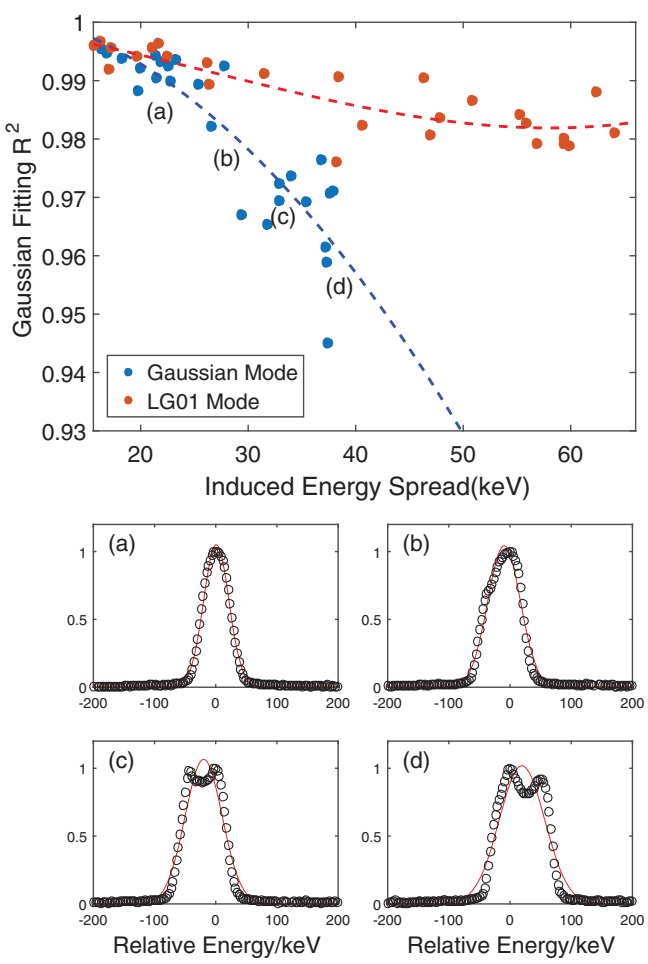

FIG. 3. Gaussian fitting coefficient of determination $R^{2}$ as a function of induced energy spread at laser heater for $\mathrm{LG}_{01}$ and Gaussian modes. (a)-(d) Four examples of energy distributions from transverse Gaussian mode laser heater with (a) 20.5, (b) 26.7 , (c) 30.1 , and (d) $37.2 \mathrm{keV}$ rms energy spread and their corresponding Gaussian fits.

first indicator of its potential for better microbunching suppression and improved FEL performance.

The second primary diagnostic (inset 4 of Fig. 1), further downstream of the linac where the $e$ beam reaches its final energy of $4 \mathrm{GeV}$, is a midinfrared (MIR) spectrometer [29], which enables characterization of microbunching at high longitudinal-space frequency from coherent secondary emission of the $e$ beam. MIR measures the coherent transient radiation from a thin film inserted into the $e$ beam and the radiation profile $I(\kappa)$ is proportional to the bunching factor of the $e$ beam [29].

$$
I(\kappa) \propto|f(\kappa)|^{2},
$$

where $\kappa$ is the spatial frequency $\kappa=1 / \lambda$ and $f(\kappa)$ is Fourier transform of longitudinal $e$-beam charge distribution. A 2D MIR spectrograph as a function of induced energy spread is shown in Fig. 4(a). The strength of differing MIR frequencies map longitudinal charge density. That is, the lower MIR frequency range components represent the $e$ beam as a whole, whereas the higher frequency components correspond to shorter longitudinal density modulations. The coherent spectral signal integrated along $k \in(3000,5000) \mathrm{cm}^{-1}$ (2-3.3 $\mu \mathrm{m}$ wavelength) is shown in red in Fig. 4(b) (Gaussian mode LH in blue for comparison). Here, a lower 

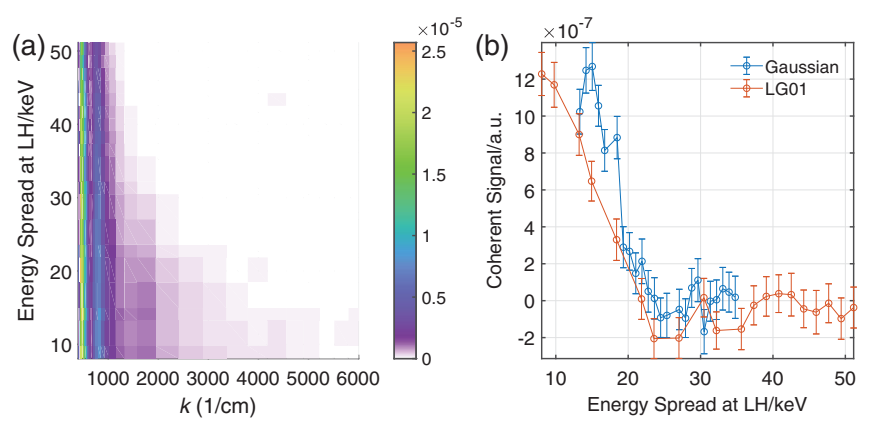

FIG. 4. (a) 2D MIR spectrograph as a function of induced energy spread by $\mathrm{LG}_{01}$ transverse mode $\mathrm{LH}$ and (b) integrated MIR spectral intensity for $k \in(3000,5000) \mathrm{cm}^{-1}$ as a function of induced energy spread by both the $\mathrm{LG}_{01}$ and Gaussian mode LHs.

spectral signal contribution signifies reduction of MBI. In the range of 15 to $20 \mathrm{keV}$ energy spread, the $\mathrm{LG}_{01}$ shows better MBI suppression compared to its Gaussian equivalent.

While effective MBI suppression can significantly improve a vast number of FEL operational modes, one of its major applications resides in increasing seeding and harmonic lasing spectral monochromaticity and brightness [3,30-32]. Theoretical and experimental analyses indicate that MBI is a primary contributor to the pedestal structure in the SXRSS spectrum $[31,33,34]$, more so than self-amplified spontaneous emission, which collectively reduce the achievable resolution in a number of ultrafast $\mathrm{x}$-ray spectroscopic techniques. In our study, the interaction of $e$ beam and $\mathrm{LG}_{01}$ laser in LH is simulated with a manually modified element LSRMDLTR in ELEGANT [35] and the SXRSS spectrum is compared using GENESIS $[27,36]$. Our startto-end simulations have shown that the $\mathrm{LG}_{01}$ mode LH could lead to $20 \%$ better monochromaticity within $1 \mathrm{eV}$ in the SXRSS spectrum [Figs. 5(c) and 5(d)]. However, a direct comparison between the two modes is not possible as the SXRSS performance changes from day to day and the machine is optimized differently each time. In our experiment, SXRSS is set at $750 \mathrm{eV}$ photon energy employing the
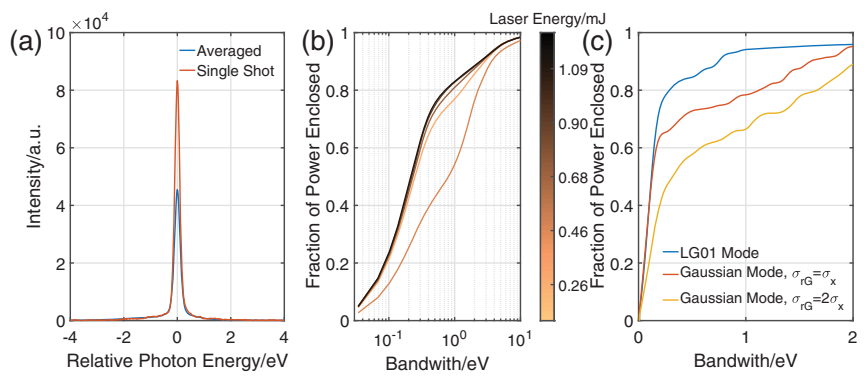

FIG. 5. (a),(b) SXRSS experiment results with $\mathrm{LG}_{01}$ mode $\mathrm{LH}$. (a) Averaged (blue line) and single shot (red line) SXRSS spectra centered at $750 \mathrm{eV}$ photon energy and (b) fraction of SXRSS spectral power as a function of bandwidth for varying $L_{01}$ mode energy in the LH. (c) Start-to-end simulation of SXRSS spectral bandwidth with Gaussian and $\mathrm{LG}_{01}$ mode laser heater at undulator length $23.1 \mathrm{~m}$.
$\mathrm{LG}_{01} \mathrm{LH}$, where numerous shots show high sideband suppression. Figure 5(a) exemplifies averaged (blue line) and a single shot spectrum (red line) in the undulator downstream of the soft x-ray seeding chicane. Figure 5(b) shows the fraction of FEL energy within certain integrated bandwidth, where the improved monochromaticity with optimal laser energy indicates that the new transverse mode laser heater works in reducing the sidebands and suppressing the microbunching.

Discussion: Transverse jitter effects.-While transverse laser jitter can have a significant impact on the induced energy distribution for most modes, it is particularly detrimental for the $\mathrm{LG}_{01}$ mode [25]. Acoustic and thermal noise can cause both pointing and transverse offset jitter in the LH pointing, thus compromising $\mathrm{LH}$ and $e$-beam spatial overlap from shot to shot, especially for the high-energy mode. Because of this jitter, the $e$ beam may occasionally overlap with the intensity minimum in the center of the laser, and other times lie along the intensity maximum at the ring. In the latter cases, the $e$ beam can experience a nearly flattop transverse laser distribution, which inevitably results in the aforementioned double-horn energy distribution.

To verify the theory and quantify the transverse jitter effects, we utilize an online camera imaging the interaction point in the LH [15] in order to nonintrusively determine the transverse position fluctuations due to pointing noise. The data are collected simultaneously with the longitudinal phase space spectrometer such that the induced energy spread can be correlated with the laser transverse jitter. We note that the $e$-beam transverse jitter is negligible compared to that of the laser. Figure 6(a) is representative of the transverse jitter with $1.7 \mathrm{~mJ}$ laser energy, which amounts to $100 \mu \mathrm{m} \mathrm{rms}$, and shows a number of consecutive central positions of the laser beam in the LH undulator. For each shot, the induced energy distribution is recorded by spectrometer. The shots of different distribution shapes are segregated in the transverse plane which indicates that correlation exists. We define the optimum position of the laser by averaging the positions of shots with $R^{2}>0.98$ and plot Gaussian fitting $R^{2}$ as a function of radial distance
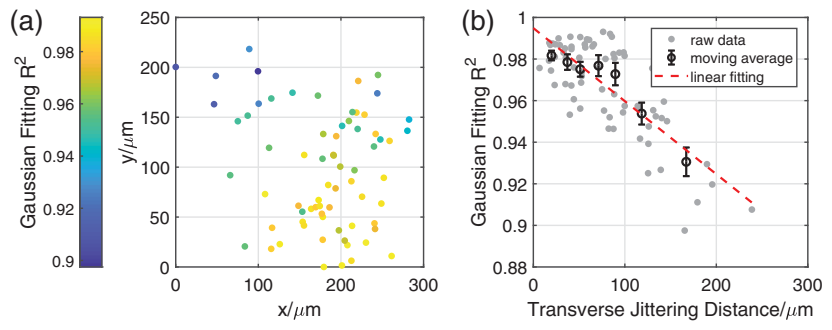

FIG. 6. Influence of transverse laser jittering for $\mathrm{LG}_{01}$ mode laser heater. (a) Positions of laser center on the camera of different shots with color representing $R^{2}$ of the corresponding laserinduced $e$-beam energy distribution. (b) Correlation between the Gaussian fitting $R^{2}$ of the energy distribution and radial distance laser jittering away from the optimal position. 
the laser jitters away from this optimum position. As shown in Fig. 6(b), correlation between the distribution structure and the jittering distance verifies our previous analyses and signifies that large transverse jittering can deteriorate the $\mathrm{LG}_{01}$ mode laser heater performance.

Conclusion.-We experimentally demonstrate that a $\mathrm{LG}_{01}$ transverse mode $\mathrm{LH}$ induces a Gaussian energy distribution of the $e$ beam over a large dynamic range and show evidence of improvement on MBI suppression compared to routine operations (i.e., Gaussian mode LH). We achieved high-efficiency optical mode conversion from a Gaussian to $\mathrm{LG}_{01}$ mode laser beam to induce adequate $e$-beam energy spread. We have also studied the impact of the $\mathrm{LG}_{01} \mathrm{LH}$ on SXRSS performance and its monochromaticity or spectral brightness. These results will fuel the next generation of engineered laser heater designs for existing and future augmented brightness linacs and $\mathrm{x}$-ray FELs.

We would like to thank and acknowledge Greg Stewart for his artwork contribution. This work was supported in part by the U.S. Department of Energy and Laboratory Directed Research and Development program at SLAC National Accelerator Laboratory, under Contract No. DEAC02-76SF00515. This material is based upon work supported by the U.S. Department of Energy, Office of Science, Office of Workforce Development for Teachers and Scientists, Office of Science Graduate Student Research (SCGSR) program. The SCGSR program is administered by the Oak Ridge Institute for Science and Education (ORISE) for the DOE. ORISE is managed by ORAU under Contract No. DE-SC0014664. All opinions expressed in this Letter are those of the authors and do not necessarily reflect the policies and views of DOE, ORAU, and ORISE.

*scarbajo@slac.stanford.edu

[1] C. Bostedt, S. Boutet, D. M. Fritz, Z. Huang, H. J. Lee, H. T. Lemke, A. Robert, W. F. Schlotter, J. J. Turner, and G. J. Williams, Rev. Mod. Phys. 88, 015007 (2016).

[2] P. Emma, R. Akre, J. Arthur, R. Bionta, C. Bostedt, J. Bozek, A. Brachmann, P. Bucksbaum, R. Coffee, F.-J. Decker et al., Nat. Photonics 4, 641 (2010).

[3] E. Allaria, D. Castronovo, P. Cinquegrana, P. Craievich, M. Dal Forno, M. Danailov, G. D'Auria, A. Demidovich, G. De Ninno, S. Di Mitri et al., Nat. Photonics 7, 913 (2013).

[4] T. Ishikawa, H. Aoyagi, T. Asaka, Y. Asano, N. Azumi, T. Bizen, H. Ego, K. Fukami, T. Fukui, Y. Furukawa et al., Nat. Photonics 6, 540 (2012).

[5] W. Ackermann, G. Asova, V. Ayvazyan, A. Azima, N. Baboi, J. Bähr, V. Balandin, B. Beutner, A. Brandt, A. Bolzmann et al., Nat. Photonics 1, 336 (2007).

[6] H.-S. Kang, C.-K. Min, H. Heo, C. Kim, H. Yang, G. Kim, I. Nam, S. Y. Baek, H.-J. Choi, G. Mun et al., Nat. Photonics 11, 708 (2017).
[7] S. Di Mitri and M. Cornacchia, Phys. Rep. 539, 1 (2014).

[8] E. L. Saldin, E. A. Schneidmiller, and M. Yurkov, Nucl. Instrum. Methods Phys. Res., Sect. A 528, 355 (2004).

[9] Z. Huang, M. Borland, P. Emma, J. Wu, C. Limborg, G. Stupakov, and J. Welch, Phys. Rev. ST Accel. Beams 7, 074401 (2004).

[10] E. L. Saldin, E. A. Schneidmiller, and M. Yurkov, Nucl. Instrum. Methods Phys. Res., Sect. A 398, 373 (1997).

[11] Z. Huang and K.-J. Kim, Phys. Rev. ST Accel. Beams 5, 074401 (2002).

[12] M. Borland, Y. Chae, P. Emma, J. Lewellen, V. Bharadwaj, W. Fawley, P. Krejcik, C. Limborg, S. Milton, H.-D. Nuhn et al., Nucl. Instrum. Methods Phys. Res., Sect. A 483, 268 (2002).

[13] E. L. Saldin, E. A. Schneidmiller, and M. Yurkov, Nucl. Instrum. Methods Phys. Res., Sect. A 490, 1 (2002).

[14] S. Heifets, G. Stupakov, and S. Krinsky, Phys. Rev. ST Accel. Beams 5, 064401 (2002).

[15] Z. Huang, A. Brachmann, F.-J. Decker, Y. Ding, D. Dowell, P. Emma, J. Frisch, S. Gilevich, G. Hays, P. Hering et al., Phys. Rev. ST Accel. Beams 13, 020703 (2010).

[16] D. Ratner, C. Behrens, Y. Ding, Z. Huang, A. Marinelli, T. Maxwell, and F. Zhou, Phys. Rev. ST Accel. Beams 18, 030704 (2015).

[17] A. Marinelli and J. B. Rosenzweig, Phys. Rev. ST Accel. Beams 13, 110703 (2010).

[18] R. Akre, D. Dowell, P. Emma, J. Frisch, S. Gilevich, G. Hays, P. Hering, R. Iverson, C. Limborg-Deprey, H. Loos et al., Phys. Rev. ST Accel. Beams 11, 030703 (2008).

[19] A. Lumpkin, R. Dejus, and N. Sereno, Phys. Rev. ST Accel. Beams 12, 040704 (2009).

[20] S. Spampinati, E. Allaria, L. Badano, S. Bassanese, S. Biedron, D. Castronovo, P. Craievich, M. Danailov, A. Demidovich, G. De Ninno et al., Phys. Rev. ST Accel. Beams 17, 120705 (2014).

[21] A. Chao and W. Chou, Reviews of Accelerator Science and Technology-Volume 7: Colliders (World Scientific Publishing Co., Singapore, 2015).

[22] C. Evain, J. Barros, A. Loulergue, M. Tordeux, R. Nagaoka, M. Labat, L. Cassinari, G. Creff, L. Manceron, J. Brubach et al., Europhys. Lett. 98, 40006 (2012).

[23] J. Lee, J.-H. Han, S. Lee, J. Hong, C. H. Kim, C. K. Min, and I. S. Ko, Nucl. Instrum. Methods Phys. Res., Sect. A 843, 39 (2017).

[24] S. Li, A. Marinelli, Z. Huang, A. Fry, J. Robinson, S. Gilevich, and D. Ratner, in Laser Heater Transverse Shaping to Improve Microbunching Suppression for X-ray FELs, Proceedings of FEL2015, Daejeon, Korea (2015).

[25] N. Liebster, J. Tang, D. Ratner, W. Liu, S. Vetter, Z. Huang, and S. Carbajo, Phys. Rev. Accel. Beams 21, 090701 (2018).

[26] L. J. P. Ament, M. Van Veenendaal, T. P. Devereaux, J. P. Hill, and J. van den Brink, Rev. Mod. Phys. 83, 705 (2011).

[27] See Supplemental Material at http://link.aps.org/supplemental/ 10.1103/PhysRevLett.124.134801 for methods: simulation and experimental apparatus, which includes Ref. [28].

[28] J. Qiang, Y. Ding, P. Emma, Z. Huang, D. Ratner, T. Raubenheimer, M. Venturini, and F. Zhou, Phys. Rev. Accel. Beams 20, 054402 (2017). 
[29] T. J. Maxwell, C. Behrens, Y. Ding, A. S. Fisher, J. Frisch, Z. Huang, and H. Loos, Phys. Rev. Lett. 111, 184801 (2013).

[30] J. Amann, W. Berg, V. Blank, F.-J. Decker, Y. Ding, P. Emma, Y. Feng, J. Frisch, D. Fritz, J. Hastings et al., Nat. Photonics 6, 693 (2012).

[31] D. Ratner, R. Abela, J. Amann, C. Behrens, D. Bohler, G. Bouchard, C. Bostedt, M. Boyes, K. Chow, D. Cocco et al., Phys. Rev. Lett. 114, 054801 (2015).

[32] Z. Zhao, D. Wang, J. Chen, Z. Chen, H. Deng, J. Ding, C. Feng, Q. Gu, M. Huang, T. Lan et al., Nat. Photonics 6, 360 (2012).
[33] Z. Zhang, R. Lindberg, W. M. Fawley, Z. Huang, J. Krzywinski, A. Lutman, G. Marcus, and A. Marinelli, Phys. Rev. Accel. Beams 19, 050701 (2016).

[34] G. Marcus, W. M. Fawley, D. Bohler, Y. Ding, Y. Feng, E. Hemsing, Z. Huang, J. Krzywinski, A. Lutman, and D. Ratner, Phys. Rev. Accel. Beams 22, 080702 (2019).

[35] M. Borland, Technical Report, Argonne National Laboratory, Report No. LS-287, 2000.

[36] S. Reiche, Nucl. Instrum. Methods Phys. Res., Sect. A 429, 243 (1999). 